\title{
Representation of Interaural Time Difference in the Central Nucleus of the Barn Owl's Inferior Colliculus
}

\author{
Hermann Wagner, Terry Takahashi, and Masakazu Konishi \\ Division of Biology, California Institute of Technology, Pasadena, California 91125
}

This paper investigates the role of the central nucleus of the barn owl's inferior colliculus in determination of the soundsource azimuth. The central nucleus contains many neurons that are sensitive to interaural time difference (ITD), the cue for azimuth in the barn owl. The response of these neurons varies in a cyclic manner with the ITD of a tone or noise burst. Response maxima recur at integer multiples of the period of the stimulating tone, or, if the stimulus is noise, at integer multiples of the period corresponding to the neuron's best frequency. Such neurons can signal, by means of their relative spike rate, the phase difference between the sounds reaching the left and right ears. Since an interaural phase difference corresponds to more than one ITD, these neurons represent ITD ambiguously. We call this phenomenon phase ambiguity.

The central nucleus is tonotopically organized and its neurons are narrowly tuned to frequency. Neurons in an array perpendicular to isofrequency laminae form a physiological and anatomical unit; only one ITD, the array-specific ITD, activates all neurons in an array at the same relative level. We, therefore, may say that, in the central nucleus, an ITD is conserved in an array of neurons. Array-specific ITDs are mapped and encompass the entire auditory space of the barn owl. Individual space-specific neurons of the external nucleus, which receive inputs from a wide range of frequency channels (Knudsen and Konishi, 1978), are selective for a unique ITD. Space-specific neurons do not show phase ambiguity when stimulated with noise (Takahashi and Konishi, 1986). Space-specific neurons receive inputs from arrays that are selective for the same ITD. The collective response of the neurons in an array may be the basis for the absence of phase ambiguity in space-specific neurons.

Barn owls obtain information about the interaural time difference (ITD) of a sound from interaural phase differences of spectral components of a sound (Moiseff and Konishi, 1981; Takahashi and Konishi, 1986). An important neural correlate for the representation of ITD is the "characteristic delay." This is de-

\footnotetext{
Received Aug. 5, 1986; revised Apr. 3, 1987; accepted Apr. 14, 1987.

We thank C. E. Carr, W. E. Sullivan, and S. F. Volman for their help throughout the work, and, in addition to them, E. I. Knudsen, C. Köppl, and G. Manley for their comments on an earlier draft of the manuscript. E. Akutagawa helped us with histology. J. Knierim and D. Bilitch instructed us on the use of the computer microscope. This work was supported by NIH Grant 14617, NSF Grant 57033, and the Max-Planck-Gesellschaft

Correspondence should be addressed to Hermann Wagner, Division of Biology 216-76, California Institute of Technology, Pasadena, CA 91125.

Copyright (C) 1987 Society for Neuroscience $0270-6474 / 87 / 103105-12 \$ 02.00 / 0$
}

fined as the ITD at which a neuron's response is independent of frequency (Rose et al., 1966). Initially, acoustic signals are processed by neurons that are narrowly tuned to frequency. If such neurons are sensitive to ITD, a similar response level recurs at ITDs separated by integral multiples of the period of the stimulating tonc (Rosc ct al., 1966; Goldberg and Brown, 1969) or, if a broadband stimulus is used, at integral multiples of the reciprocal of the neuron's best frequency (Geisler et al., 1969). Neurons narrowly tuned to frequency may exhibit a characteristic delay, but since the neurons have multiple response maxima even to stimulation with noise, they cannot signal, by means of their relative spike rate, their characteristic delay to the next neural station. Thus, it is important to discriminate between what a neuron can "exhibit" and what it can "signal." Neurons narrowly tuned to frequency may be said to signal an interaural phase difference. We call the inability of a neuron to signal a unique ITD "phase ambiguity."

Neurons in the external nucleus of the owl's inferior colliculus ("external nucleus") respond only to sounds emanating from a restricted direction (space-specific neurons) and form a map of auditory space (Knudsen and Konishi, 1977, 1978). These neurons receive input from a wide range of frequencics $\left(Q_{10 \mathrm{~dB}}=1-\right.$ 4; Knudsen, 1984) and do not show phase ambiguity if stimulated with noise (Takahashi and Konishi, 1986). Neurons in the central nucleus of the inferior colliculus ("central nucleus") that are sensitive to ITDs have been found in the barn owl (Takahashi and Konishi, 1983), as well as in mammals (Rose et al., 1966; Geisler et al., 1969; Stillman, 1971; Yin and Kuwada, 1983, 1984). These neurons, in contrast to space-specific neurons, show phase ambiguity, are arranged tonotopically, and are narrowly tuned to frequency $\left(Q_{10 \mathrm{~dB}}=1-14 ;\right.$ Knudsen, 1984) (barn owl: Knudsen and Konishi, 1978; Takahashi and Konishi, 1983; cat: Rose et al., 1963, 1966; Geisler et al., 1969; Merzenich and Reid, 1974; Roth et al., 1978; Semple and Aitkin, 1979). Retrograde tracing studies by Knudsen $(1983,1984)$ indicate that a space-specific neuron receives inputs from many isofrequency laminae of the central nucleus.

We investigated how the functional organization of the central nucleus and its projection onto the external nucleus subserve the resolution of phase ambiguity, and present evidence for (1) a representation of a single ITD in arrays of neurons that are oriented perpendicularly to isofrequency laminae of the central nucleus, (2) a projection from arrays in the central nucleus to restricted loci (space-specific neurons) in the external nucleus, and (3) a 2-dimcnsional map of the interaural phase difference in the central nucleus that covaries along one dimension with the frequency map to create the map of a third, derived, variable, a map of ITD. 


\section{Materials and Methods}

Surgery. Data were collected from 24 barn owls (Tvto alba). Animals were anesthetized with ketamine $(4 \mathrm{mg} / \mathrm{kg} / \mathrm{hr})$ and placed in a stereotaxic head-holder that fixed the head such that the plane defined by the center of the ear bars and the ventral surface of the palatine ridge was tilted $45^{\circ}$ downward from the horizontal plane. A stainless steel head-plate was cemented to the skull, and a reference post was glued onto the skull at the intersection of the interaural axis and the midline of the skull. The head-holder was removed after the cement had dried and part of the dural surface was exposed through a craniotomy. A heating pad warmed the animal throughout an experiment. Glass-coated platinumiridium electrodes were positioned with respect to the reference post and advanced through the brain from dorsal to ventral with a remotecontrolled stepping motor. Whenever a bird was not killed immediately after an experiment, the following measures werc taken: with the use of clean surgical tools. the craniotomy and skin incisions were closed, a topical antibiotic ointment was applied, a separate room for individual caging was provided and the bird was inspected daily. Food consumption, which usually returned to the normal level within $24 \mathrm{hr}$ after surgery, served as a sensitive indicator of the bird's health. Birds that did not resume feeding within a $24 \mathrm{hr}$ period received injections of glucose and saline until they began to cat normally. All of the above treatments, taken together reduced discomfort and effectively prevented sickness and infection.

Stimuli. All experiments were conducted in an anechoic soundproof chamber. Sound was delivered through earphones placed in the external auditory meatus (Moiseff and Konishi, 1981). Stimuli consisted of 100 msec bursts ( $5 \mathrm{msec}$ rise/fall time) of cither noise (using a customdesigned noise generator with a nearly flat spectrum between 3 and 9 $\mathrm{kH} z$, as measured by cochlear microphonic potentials) or tones (General Radio 1310B) presented at a level 10-40 dB above the neurons' threshold. The frequency of the tones was chosen to lie within the excitatory tuning curve of the units. Usually, a frequency near the best frequency was used. Stimuli were repeated at a rate of $1.5 / \mathrm{sec}$. A digital delay circuit (Moiseff and Konishi, 1981) controlled ITD ( $\pm 300 \mu \mathrm{sec}$ range; sampling interval usually $30 \mu \mathrm{scc}$ ).

Data collection. Multi-unit and single-unit responses were recorded extracellularly in the inferior colliculus. Responses were expressed as the number of spikes to 5 or 10 stimulus repetitions for each ITD. Responses at different ITDs were combined into a so-called ITD response function and normalized with respect to the maximal response. Thus, the term "relative response level" means the percentage of response with respect to the maximal response. Yin et al. (1986) have reported that neurons respond at similar levels to ITD intervals that are related to the period of each neuron's best frequency rather than to that of its characteristic frequency. Accordingly, we used best frequencies and not characteristic frequencies for the analysis. The best frequency of a neuron to binaural stimulation was determined at the ITD that yielded the best response upon listening to an audiomonitor and observing the number of spikes on an oscilloscope. Use of visual and auditory monitoring is adequate for determining the best frequency (see also Kiang, 1965).

Earphone calibration. We measured the phase response of the earphones with a calibrated microphone $(1 / 2$ in. $B \& K)$ over a range from 400 to $10.000 \mathrm{~Hz}$ in steps of $160 \mathrm{~Hz}$. Furthermore, while recording from a unit ( 75 cases), we first obtained an ITD response function, reversed the carphones, and obtained a second ITD response function. The mean interaural phase values (see below) of these 2 ITD response functions were subtracted from each other and the difference divided by 2 . From the phase-difference values obtained in this way, and from those obtained from the calibration experiment, we compiled a correction table and corrected the ITD values read from the delay circuit accordingly. The 2 types of measurements agreed to within \pm 0.03 cycles. The variability found in experiments with different owls was well below the neurons' selectivity for ITDs. Since the ITD sensitivity of the neurons is independent of stimulus intensity (Moiseff and Konishi, 1981; Takahashi et al., 1984), the interaural intensity difference was simply set to 0 in most of the experiments.

IIistology: Nissl stains. Electrolytic lesions ( $-2 \mu \mathrm{A} \mathrm{DC} ; 10 \mathrm{sec})$ were made for the identification of recording sites. Following a survival period of 2-8 d, birds were killed with an overdose of sodium pentobarbital, transcardially exsanguinated with saline, and fixed by perfusion with $10 \%$ Formol-saline. Brains were blocked, removed, infiltrated with $30 \%$ sucrose, sectioned $(30 \mu \mathrm{m})$ in the coronal plane, and stained with cresyl violet.

HRP. Crystals of HRP were dissolved in Tris-buffered saline ( $\mathrm{pH} \mathrm{8.3)}$ to a concentration of $7-10 \%$. The HRP solution was drawn into a glass micropipette with an inner diameter of $15-20 \mu \mathrm{m}$ at the tip. Single ncuron responses, which could be recorded through the HRP pipette, guided placement in the external nucleus. All neurons in a dorsoventral penetration were selective for the same ITD. Typically, 2-4 neurons were recorded in a single penetration. When the desired area was located we applied $\mathrm{a}+2 \mu \mathrm{A}$ pulsed DC current $(5 \mathrm{sec}$ on, $5 \mathrm{sec}$ off) for $10 \mathrm{~min}$ and iontophoretically deposited HRP at a single location in the vicinity of the recording sites. After a 2 d survival period, the owl was anesthetized with sodium pentobarbital, transcardially exsanguinated with saline, and fixed by perfusion with a mixture of $1 \%$ paraformaldehyde and $1.25 \%$ glutaraldehyde in phosphate buffer $(0.1 \mathrm{M}, \mathrm{pH} 7.4)$, followed by $10 \%$ sucrose in phosphate buffer. Brains were removed, infiltrated with $20 \%$ sucrose, and $30 \mu \mathrm{m}$ frozen sections collected in ice-cold buffer Sections were treated for HRP histochemistry according to the protocol of Mesulam (1978), using tetramethyl benzidine as the chromagen. Sections were counterstained with neutral red and analyzed under a light microscope using dark- and bright-field optics.

Data analysis. Circular statistics (Batschelet, 1981) are used for the analysis of the data. First. an ITD is converted into interaural phase difference $\Phi$ by dividing the ITD by the period of the stimulus tone or, in the case of a noise stimulus, by the period of the neuron's best frequency. The integer part of the result is disregarded in the further calculation. If the ITD is less than zero, 1 is added to the phase value. The response of a neuron at the $i$ th ITD is thus a vector having the direction $\Phi$, and magnitude $A$, the normalized spike count. The selectivity of a ncuron for a particular interaural phase difference is calculated as vector strength VS (Goldberg and Brown, 1969). The direction of the mean vector, the mean interaural phase $\bar{\phi}$, denotes the phase value around which the response is centered. The circular standard deviation $s_{i}$ can be derived from vector strength $V S$ :

$$
s_{\mathrm{c}}=(180 / \pi) \cdot \sqrt{2 \cdot(1-V S)} .
$$

Yin and Kuwada (1983) published a method for the calculation of a characteristic delay. They stimulated a neuron with different frequencies and tested whether the mean interaural phase of the response and the stimulus frequency were linearly related. The level of significance was read from a table composed of the "mean square error" (mse). In contrast to the correlation coefficient, mse is independent of the slope of the regression line. The mean square error is a measure of the deviation of the data points from the regression line in the $y$-direction and is defined as follows:

$$
m s e=\sum\left(\tilde{\Phi}_{i}-a-b * f_{i}\right)^{2} /(N-2) .
$$

$\bar{\Phi}_{t}$ and $f_{t}$ are, respectively, the actual mean interaural phase and frequency values, and $a$ and $b$ are the intercept and slope of the regression line, respectively. $N-2$ are the degrees of freedom. The slope of the linear regression line has units of time and represents the characteristic delay of the neuron, whereas the $y$-intercept, called characteristic phase, has units of cycles and represents the phase angle at which the characteristic delay occurs relative to the maximum response. Values around zero (or 1.0 ) indicate a characteristic delay near the maximum discharge, whereas values around 0.5 indicate a characteristic delay near the minimum discharge of the neuron. Summing up the spike counts at a particular ITD to stimulation with different frequencies yields the "composite response" of a unit (Yin and Kuwada, 1983). The composite peak denotes the highest response counted for different ITDs.

\section{Results}

\section{General properties of ITD-sensitive neurons in the central nucleus}

The response of neurons in the central nucleus varies in a cyclic manner with the ITD of a sound stimulus. The period of the ITD response function is similar to that of the stimulus tone (broken lines in Fig. 1 $A$ ) or, for noise stimuli, to that of the neuron's best frequency (solid lines in Fig. $1 A$ ). The mean absolute difference between the period of the stimulating tone and 

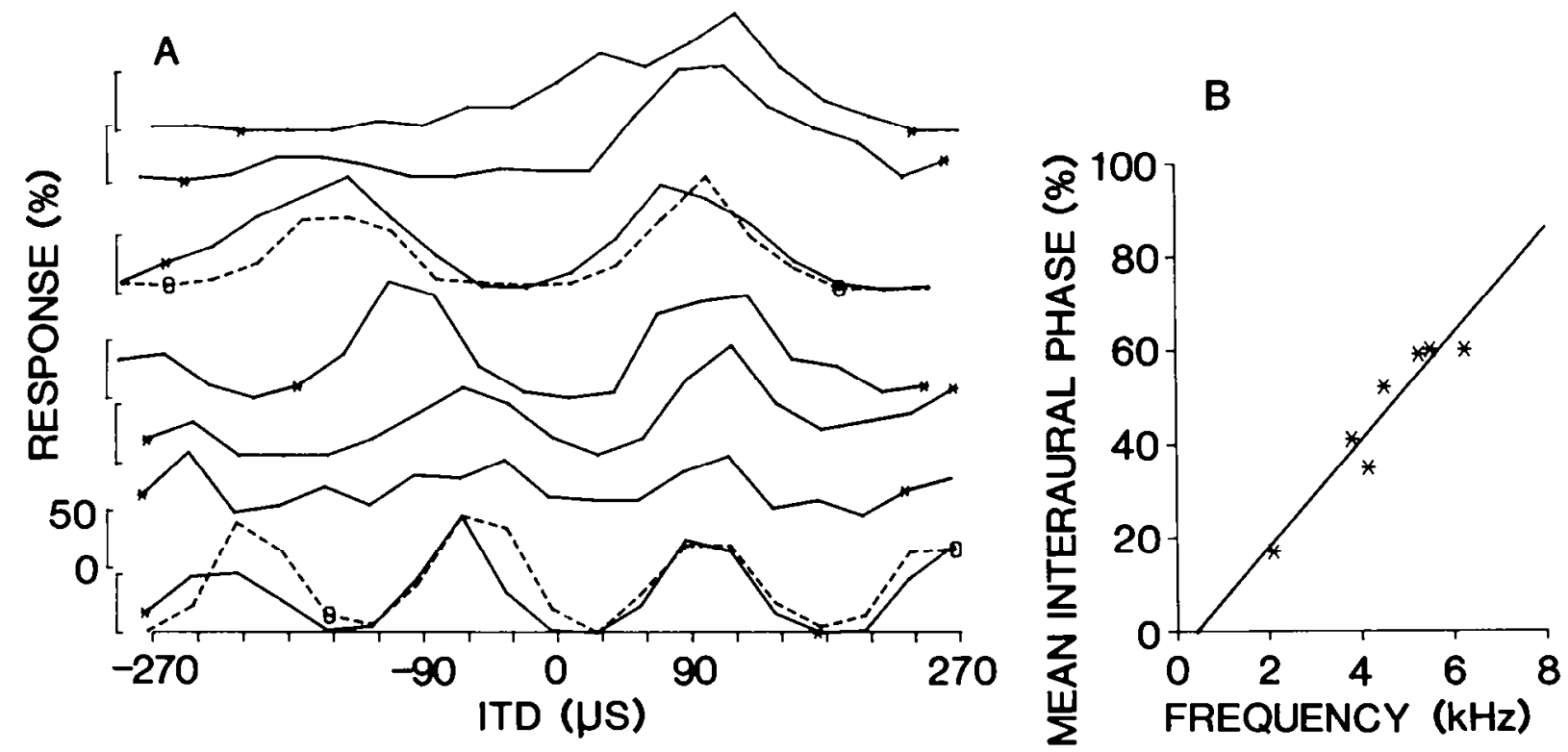

Figure 1. Neurons in one penetration form an array that exhibits an ITD. A, Normalized response curves from 7 recording sites in the left inferior colliculus within a frequency range of $2100-6700 \mathrm{~Hz}$. Solid lines refer to stimulation with noise, broken lines to stimulation with tones. The asterisks and circles delimit the range of ITD values used for the calculation of mean interaural phase $\bar{\Phi}$ and vector strength for noise and tone stimuli, respectively. These parameters were calculated over an integral number of cycles, usually with start and end points near a response minimum. The sampling interval between 2 adjacent ITDs is $30 \mu \mathrm{sec}$. Negative ITDs indicate that the left signal is leading in time. $B$, Plot of $\bar{\Phi}$ as a function of best frequency for the individual recording sites. The mean square error is $0.002(p<0.005)$. Slope $(113 \mu$ sec) and $y$-intercept $(-0.05$ cycles) show that the array-specific ITD corresponds to an ITD at which all neurons of the array respond maximally. For quantification of parameters, see Table 1.

the period of the ITD response function is $6.6 \pm 8.2 \mu \mathrm{sec}(42$ cases). The mean absolute difference between the period of the neuron's best frequency and the period of the ITD response function to stimulation with noise is $14.0 \pm 9.8 \mu \mathrm{sec}$ ( 42 cases). Generally, all response peaks of any given ITD response function have similar heights and occur at the same interaural phase difference, which is measured by mean interaural phase (Figs. $1 A, 6 A)$. Thus, ITD-sensitive neurons in the central nucleus show phase ambiguity. Selectivity to ITD is found in neurons tuned to best frequencies ranging from $500 \mathrm{~Hz}$ (lower frequencies were not tested) to $9500 \mathrm{~Hz}$. The ITD response functions obtained from stimulation with noise are equivalent to those obtained from stimulation with the neurons' best frequencies: similar values of vector strength and mean interaural phase are measurcd, although the absolute discharge level under these 2 stimulus conditions often differs.

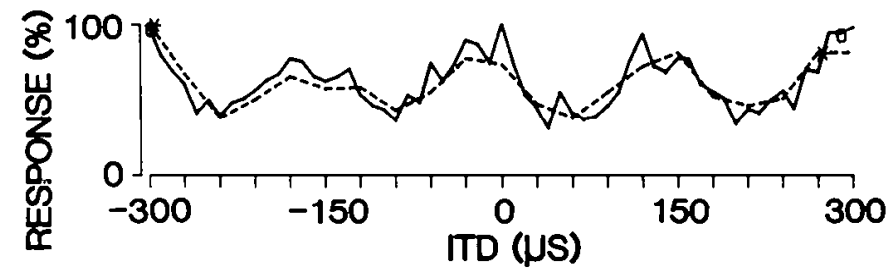

Figure 2. Comparison of the response curves of a multi-unit recording obtained with $10 \mu \mathrm{sec}$ (solid line, $\mathrm{O}, \mathrm{VS}=0.17 ; \overline{\boldsymbol{\Phi}}=0.92$ ) and with 30 $\mu$ sec sampling intervals (broken line, ${ }^{*}$, VS $=0.18, \bar{\Phi}=0.90$ ). Stimulus frequency was $6667 \mathrm{~Hz}$, a frequency that yielded at very high response at this recording site. Other conventions as in Figure 1 . Statistical parameters can be reliably calculated from data using a $30 \mu \mathrm{sec}$ sampling interval.
The vector strength of the ITD response function obtained at a location having a best frequency of $5500 \mathrm{~Hz}$, shown in Figure $1 \mathrm{~A}$, is 0.11 . The circular standard deviation for the mean intcraural phase is \pm 0.21 cycles or $\pm 38 \mu \mathrm{sec}$. In this curve, the lowest response level relative to the maximum is $48 \%$. Response curves with lower vector strengths are flatter, and mean interaural phase becomes even less well defined. We arbitrarily chose

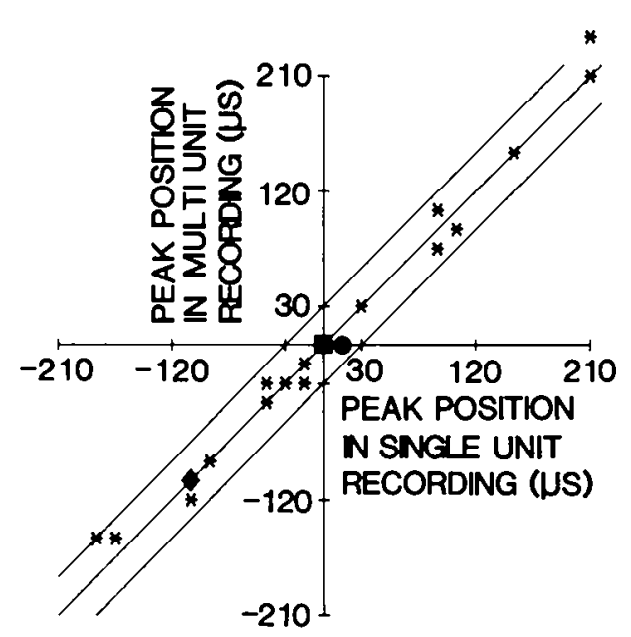

Figure 3. Comparison of response peaks from multi-unit clusters with those from single units. Symbols $(\mathbf{0}, \boldsymbol{\varphi}, *)$ represent $5,3,2$, and 1 data points at a particular locus, respectively. The total number of points is 26 . If the values were equal, they would lie on the middle diagonal line, which indicates $45^{\circ}$ angle. The other diagonal lines are offset by $30 \mu \mathrm{sec}$ with respect to the middle line and indicate the sampling interval of the measurements. For further explanations, see text. The peaks of multi-unit clusters and those of corresponding single units always lie within one sampling interval. 


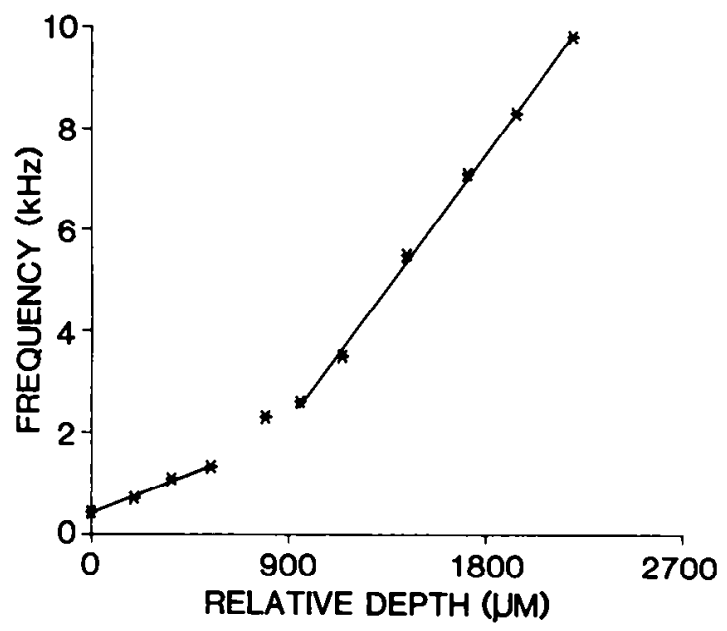

Figure 4. Tonotopy in the central nucleus. The best frequency of ncurons increases monotonically with depth in a dorsoventral penetration. In some parts of the nucleus, there are 2 regions with different linear rclationships between spatial coordinate and best frequency. A linear regression was calculated for recording sites $1-4$ and another for recording sites 6-11 from the top of the nucleus. Both correlation coefficients are significantly different from zero $(p<0.05)$. The slopes are $1685 \mathrm{~Hz} / \mathrm{mm}$ for the low-frequency region and $5845 \mathrm{~Hz} / \mathrm{mm}$ for the high-frequency region. The fourth, sixth, seventh, and eighth recording sites from the top of the nucleus represent single-unit recordings; all others represent multi-unit clusters. Relative depth refers to the depth from the top of the nucleus.

0.1 as a threshold and did not include ITD response functions with vector strengths of less than 0.1 in the analysis. The tuning of neurons in the central nucleus to ITDs, as measured by (1) circular standard deviation and (2) half-maximal width, is between 20 and $60 \mu \mathrm{sec}$ (Figs. 1, 6).

Since ITD response functions are almost symmetrical around the response maximum, mean interaural phase can be calculated reliably from a few points. Generally, a sampling interval of 30 $\mu$ sec was used in the recordings. Controls at a finer sampling interval $(10 \mu \mathrm{sec})$ revealed no differences in mean interaural phase (Fig. 2; 49 cases, mean of absolute difference $=0.007 \pm$ 0.008 cycles).

The data presented here include multi-unit and single-unit recordings. We found no conspicuous differences in vector strength or mean interaural phase between the 2 types of recording (Figs. 1, 6). Furthermore, response peaks of single-unit and multi-unit recordings encountered in one penetration at distances not farther apart than $150 \mu \mathrm{m}$ occur at similar ITDs. In Figure 3 we compare the response peaks of such pairs and use the ITDs of the actual peaks and not the values of the mean interaural phase, since mean interaural phase depends on frequency, and frequency changes with depth in the central nucleus (see below). The responses at 5500 and $6250 \mathrm{~Hz}$ shown in Figure 1.4 represent one of the 26 points plotted in Figure 3, which were taken from 22 penetrations and 10 owls. In all 26 cases, the difference between the peaks lies within $30 \mu \mathrm{sec}$, or one sampling interval.

\section{ITD specific to an array of neurons}

Individual neurons in the central nucleus show phase ambiguity. As the central nucleus is a tonotopic structure (Knudsen and Konishi, 1978), we hypothesized that a group of neurons from a penetration, which would encompass a broad band of frequencies, would exhibit a single ITD. A test of this idea requires

\begin{tabular}{|c|c|c|c|c|c|}
\hline Site & $\begin{array}{l}\text { Recording } \\
\text { depth } \\
(\mu \mathrm{m})\end{array}$ & Type & $\begin{array}{l}\text { Best } \\
\text { frequency } \\
(\mathrm{Hz})\end{array}$ & $\begin{array}{l}\text { Mean } \\
\text { interaural } \\
\text { phase } \\
\text { (cycles) }\end{array}$ & $\begin{array}{l}\text { Vector } \\
\text { strength }\end{array}$ \\
\hline 1 & 15,170 & $\mathbf{M}$ & 2100 & 0.17 & 0.65 \\
\hline 2 & 15.860 & $S$ & 3800 & 0.41 & 0.40 \\
\hline 3 & 15,920 & S & $4160(4167)$ & $0.35(0.39)$ & $0.44(0.55)$ \\
\hline 4 & 16,070 & S & 4500 & 0.52 & 0.57 \\
\hline 5 & 16,270 & S & 5250 & 0.59 & 0.32 \\
\hline 6 & 16,390 & $\mathbf{M}$ & 5500 & 0.60 & 0.11 \\
\hline 7 & 16,530 & $S$ & $6250(6687)$ & $0.60(0.66)$ & $0.60(0.51)$ \\
\hline
\end{tabular}

Parameters are listed from top to bottom. M, multi-unit clusters; S, single-uni recordings. Values in parentheses refer to stimulation with tones.

3 steps: First, one must find a penetration angle that comprises a broad band of frequencies. Second, it must be demonstrated that an ITD exists that activates all neurons in a penetration at the same relative response level. Third, a functional significance has to be assigned to this ITD.

Dorsoventral penetrations fulfill the first condition. Singleunit and multi-unit data reveal a monotonic increase in the best frequency of ncurons from dorsal to ventral (Fig. 4; see also Figs. $1 A, 6 A$, Table 1). The penetration shown in Figure 4, which is from the center of the nucleus, encompasses frequencies ranging from 500 to $10,000 \mathrm{~Hz}$. Gencrally, the frequency ranges were larger than $5000 \mathrm{~Hz}$. As indicated by the 2 different regression lines of Figure 4, the slope for frequencies lower than 1500 $\mathrm{Hz}$ may be different from that for frequencies higher than 2000 $2500 \mathrm{~Hz}$. Our analysis will be restricted to the high-frequency range, which is most important for sound localization in the barn owl (Konishi, 1973).

The test of the second condition is based on the following relationship: If an ITD exists that activates all units in a penetration at the same relative response level, mean interaural phase will be a linear function of best frequency. ITD response functions obtained in a dorsoventral penetration within a frequency range of $2100-6700 \mathrm{~Hz}$ are shown in Figure $1 \mathrm{~A}$. An outstanding feature of Figure $1 A$ is the common response peak at about $+90 /+120 \mu \mathrm{sec}$. In Figure $1 B$, mean interaural phase is plotted as a function of the best frequency for the recording sites shown in Figure $1 A$ and quantified in Table 1. Regression and correlation analyses demonstrate that the variables are linearly related ( $m s e=0.002 ; p<0.005$ ). The value of the slope, $113 \mu \mathrm{sec}$, corresponds to the ITD at which all units are activated at the same relative response level. This valuc agrees well with what one would expect from an inspection of Figure $1 A$. The $y$-intercept of the regression line, -0.05 cycles, confirms that all units are maximally activated at the ITD represented by the slope of the regression line.

In this study we made 231 penetrations through the central nucleus. We shall refer to all the neurons recorded in one penetration as an "array." An "array-specific ITD" is the ITD that activates all the neurons of an array at the same relative response level. In linear-regression analysis, the reliability of the results depends on the number of sample points. If the number of recording sites is small, even minor variations in the responses of individual neurons have large influences on the slope and intercept of the regression line defining the properties of an array. Therefore, we evaluated only the 93 penetrations in which 4 or 

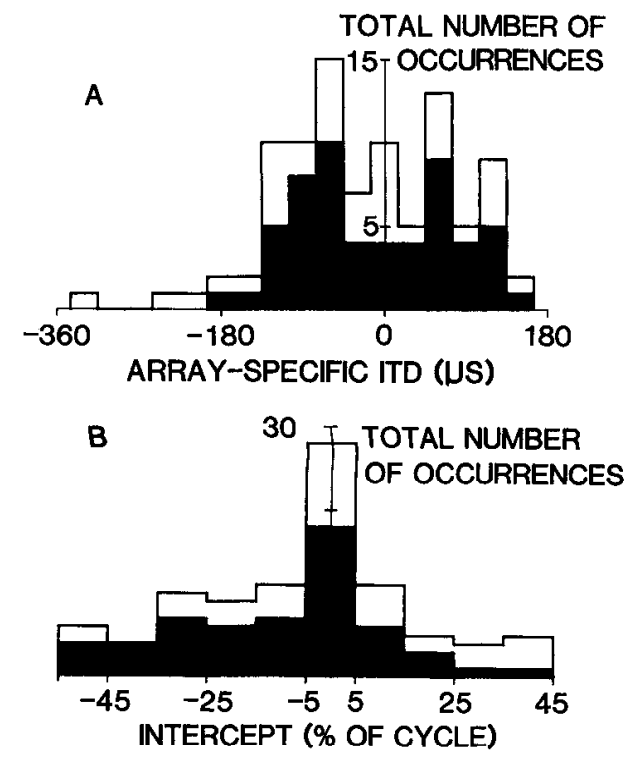

Figure 5. Histogram of slopes ( $A$, array-specific ITD) and intercepts $(B)$ of the regression lines characterizing arrays. The filled areas show the 57 values for which the linearity test indicates a probability of randomness of less than 0.05 . The data of all 93 penetrations are plotted within the open bars. Bin width is $30 \mu \mathrm{sec}$ in $A$ and $10 \%$ of a cycle in $B$. All ITDs seem to be equally represented within the barn owl's physiological ITD range. Intercepts around 0.0 are most common, indicating that in many cases the maximum response at the individual recording sites contributes to the array-specific ITD.

more recording sites sensitive to ITDs could be obtained. Fiftyseven penetrations $(61 \%)$ yielded statistically significant values of mean square error $(p<0.05)$. Array-specific ITDs range from -326 to $153 \mu \mathrm{sec}$ (Fig. $5 A$ ), but only 4 of the 93 values were greater than the physiological ITD range of the barn owl [about $\pm 180 \mu \mathrm{sec}$ (Moiseff and Konishi, 1981); note that in Fig. 5A this observation is obscured by the fact that the important bin ranges from -195 to $-165 \mu$ sec]. All ITDs seem to be equally represented within this range. The distribution of the $y$-intercepts has a peak around zero (Fig. $5 B$ ). Thus, in a large proportion of the arrays, the array-specific ITD elicits a maximal response in all neurons forming the array. Fifty-four percent of the $y$-intercepts lie within $0.0 \pm 0.15$ cycles, whereas only $16 \%$ lie between $0.5 \pm 0.15$ cycles. The filled areas in Figure 5 represent data from penetrations with significant mean square errors. The addition of values from penetrations that did not meet the significance criterion does not change the shape of the distributions shown in Figure 5. Note, however, that the array-specific ITDs that lie outside the physiological ITD range of the barn owl are all from arrays that did not meet the significance criterion.

\section{The characteristic delay of single neurons in an array}

To compare the array-specific ITD with the characteristic delays of the individual neurons forming an array, we attempted to determine these delays. Since the characteristic delay of a neuron is also calculated by linear-regression analysis, the constraints mentioned in the last paragraph apply: the fewer the number of frequencies that can be tested, the less reliable are the results. Also, the test frequencies should be spaced in intervals that are related to the ITD selectivity of the neuron that is investigated. We measured ITD selectivity by circular standard deviation. A typical circular standard deviation for a neuron in the central nucleus was $30 \mu \mathrm{sec}$. Therefore, we chose test frequencies whose periods differed by $30 \mu \mathrm{sec}$. Only 13 neurons could be tested with 4 or more test frequencies differing in period by $30 \mu \mathrm{sec}$. All of these neurons had best frequencies below $5500 \mathrm{~Hz}$ and were from different arrays. High-frequency units (best frequency $>7500 \mathrm{~Hz}$ ) could usually be tested with only 2 frequencies that differed in period by $30 \mu \mathrm{sec}$. Therefore, for most of the arrays, the array-specific ITD and the characteristic delay of individual neurons could not be compared.

Eleven of the 13 neurons showed a significant linear relationship between mean interaural phase and stimulus frequency (nonrandom mse; $p<0.05$ ). Thus about $80 \%$ of the neurons tested exhibited a characteristic delay. In 7 cases the characteristic delay of a unit could be compared with the ITD exhibited by the array to which the unit belonged. The mean absolute difference between the array-specific ITD and the characteristic delay of a unit belonging to that array was $84 \mu \mathrm{sec}$ for the 7 cases. We will comment more on these problems in the Discussion.

\section{Relationship between arrays and space-specific neurons}

In the third step of testing our hypothesis, we used the retrograde transport of HRP injected into a restricted area of the external nucleus to study the relationship between arrays of neurons in the central nucleus and the injection site. Cells in the external nucleus, unlike those in the central nucleus, are selective for a unique ITD (Takahashi and Konishi, 1986). We show the data from one representative owl in Figure 6 and summarize the results of 4 such experiments in Figure 7.

First, the ITD selectivity at an injection site in the external nucleus was determined (Fig. $6 A$, uppermost response curve). Then, an array in the central nucleus, exhibiting an ITD similar to that found at the injection site in the external nucleus, was marked by small, electrolytic lesions (Fig. 6, $A, B$ ). Most of the labeled cells were found in the central nucleus. Labeling also occurred in the external nucleus, ipsi- and contralaterally. In Figure $6 \mathrm{~A}$ we show the somata labeled in the inferior colliculus ipsilateral to the injection site. The density of labeled somata in sections near the lesions $( \pm 150 \mu \mathrm{m})$ is much higher than that in the sections farther away from the lesioned track (Fig. 6A). Thus, space-specific neurons in the external nucleus receive inputs from an array in the central nucleus that is selective for the same ITD as the target neurons. Labeled somata are concentrated around and lateral to the lesioned track. This distribution is consistent with the observation that the same ITD is exhibited over a range of about $500 \mu \mathrm{m}$ in the mediolateral direction in all but the most anterior part of the central nucleus (for changes of the array-specific ITD in the anteroposterior direction, see next section). In order to rule out the possibility that lesions per se produced a label-like artifact within somata, we placed lesions above and below the central nucleus in one animal. In this owl, labeled somata are found within the nucleus and not at the lesion sites.

Injections were made at different locations of the external nucleus in 4 experiments to study the projection of the central nucleus onto the external nucleus (Fig. 7). In one of these experiments, the tracer spread into the central nucleus. We included the data of this experiment in Figure 7 because the distribution of cells labeled in the central nucleus was not affected by this spread. Labeled somata occur along the whole anteroposterior axis of the central nucleus. However, each distribution 

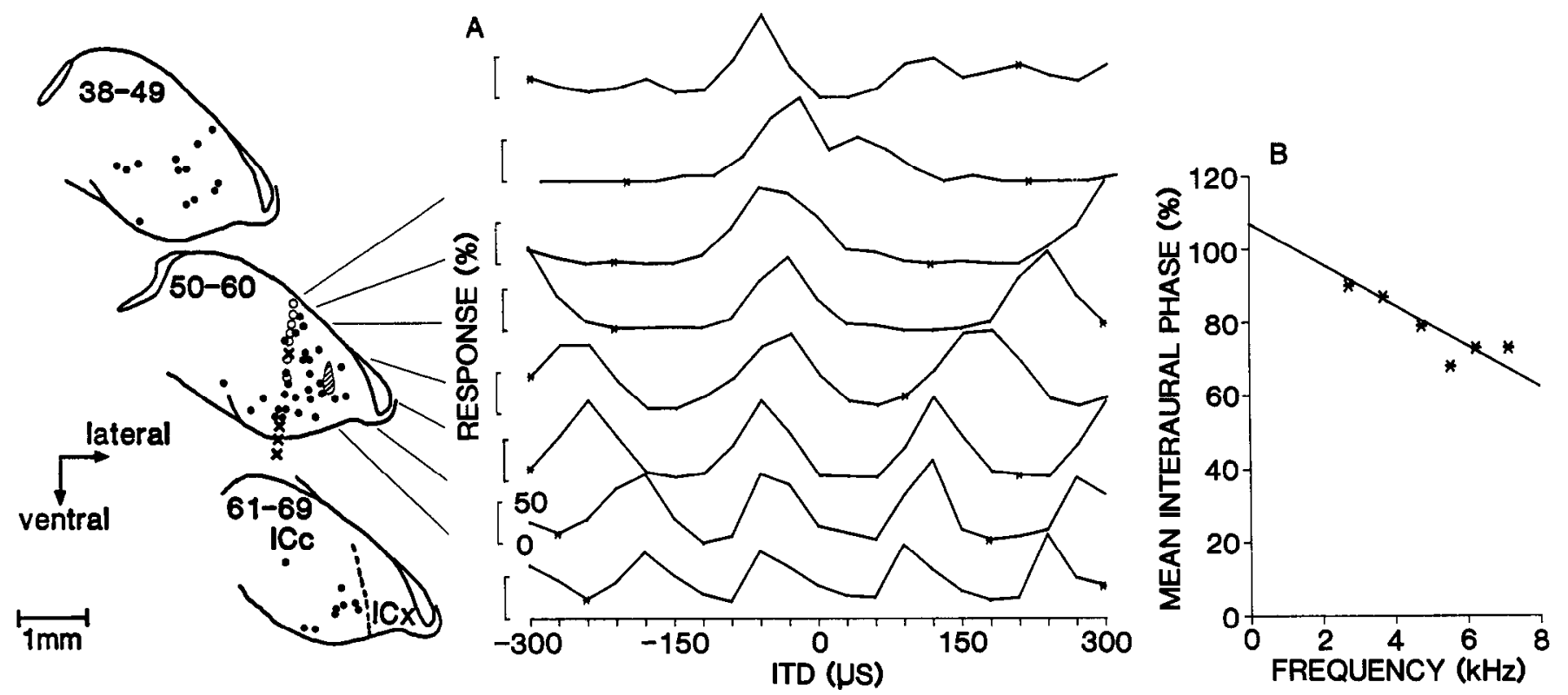

Figure 6. The connection between neuronal arrays in the central nucleus and a small locus in the external nucleus. $A$, Middle panel shows the response curves obtained at the recording sites in the right central nucleus. The uppermost curve was recorded in the same penetration where the tracer was injected, but $405 \mu \mathrm{m}$ dorsal to the injection site. Stimuli were tones at the best frequencies (central nucleus recordings) or noise (external nucleus recording). The sampling interval was $30 \mu \mathrm{sec}$. Other conventions are as in Figure 1 . The left schematic drawing shows 3 coronal sections of the central nucleus. Labeled somata $(0)$, lesions $(\times)$, recording sites $(0)$, and the injection site (striped area) are shown. Numbers in the drawings refer to the identification numbers of $30 \mu \mathrm{m}$ sections, superimposed for the drawings. The outlines of sections 44,55 , and 65 are drawn. Higher numbers belong to more anterior sections. $I C x$, External nucleus; $I C c$, central nucleus. $B$, Array-specific ITD is $-57 \mu$ sec $(m s e=0.0021)(p<$ 0.005 ). The response peak of the curve recorded near the injection site is at $-60 \mu \mathrm{sec}$. All recordings, except that near the injection site, were from multi-unit clusters.

shows a clear peak, and the distribution of labeled somata in the central nucleus varies systematically with the ITD represented at the injection site in the external nucleus: the more contralateral the preferred ITD in the external nucleus, the more posterior is the maximum of the distribution in the central nucleus. This provides anatomical evidence for a systematic mapping of ITDs within the central nucleus.

\section{Maps of time cues in the central nucleus}

We gathered enough data from 4 owls to evaluate the dependence of mean interaural phase on position within the nucleus. Figures 8-11 summarize the results obtained from one representative owl.

Seven penetrations (P1-P7) were made systematically in the central nucleus (Fig. $8 A$ ). The ITD response functions at 4-8 sites per penetration were recorded. The posteromedial part, which contains neurons insensitive to ITD, was not investigated (Fig. 8A). Where possible, data were taken at locations having preselected best frequencies $(3333,3704,4167,4762,5555$, 6667 , and $8333 \mathrm{~Hz}$ ) to evaluate the distribution of mean interaural phase within these isofrequency laminae. Four of the 7 penetrations (P1, P4, P5, P7) were marked by lesions. The lesions and the electrode track of a fifth penetration (P3) were identified histologically (Fig. 8B). Therefore, the locations of 5 penetrations are precisely known. Only the data from these penetrations were used for constructing the maps shown in Figures 10 and 11.

Array-specific ITDs change with the position of the penetration within the central nucleus (Fig. 9). Only units with best frequencies below $7500 \mathrm{~Hz}$ were used for the analysis, because mean interaural phase values of the $8333 \mathrm{~Hz}$ isofrequency lam- ina were displaced with respect to the data from all other isofrequency laminae (Fig. 11). P2 exhibits the most negative ITD and P7 the most positive. Array-specific ITDs increase monotonically from P3 to P7. Although the positions of P2 and P6 could not be verified histologically, the curves obtained from these penetrations are included in Figure 9. The array-specific ITD of P2 indicates that this penetration lies close to P1. The

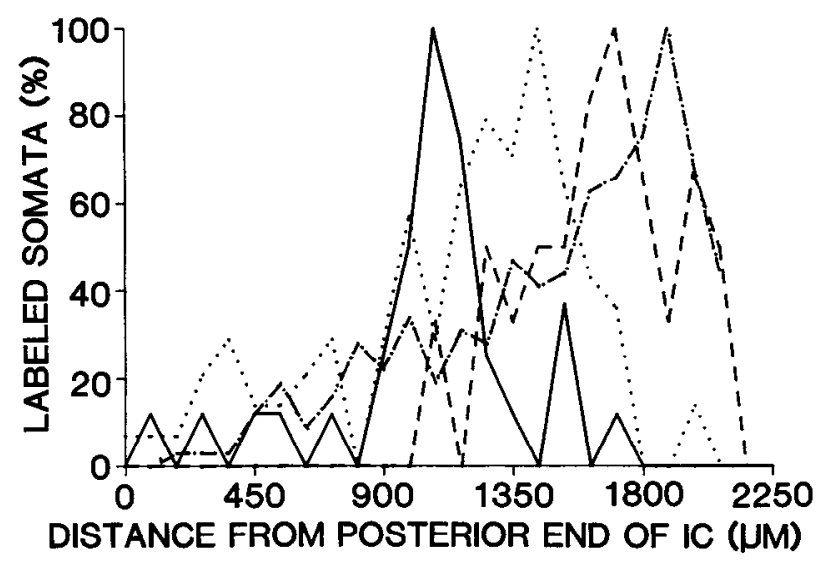

Figure 7. The ITD represented at the injection site in the external nucleus and the maximal number of the labeled somata in the central nucleus covary. The peak of the ITD response curve near the injection site and the total number of labeled somata in each curve are as follows: (-), $120 \mu \mathrm{sec}$ contralateral (33); ( . . ), $90 \mu \mathrm{sec}$ contralateral (103); $(--), 60 \mu \mathrm{sec}$ contralateral (37); (- - ) , $15 \mu \mathrm{sec}$ ipsilateral (227). Notice that, in contrast to Figure 6 , only labeled somata in the central nucleus are considered. The posterior end of the inferior colliculus $(I C)$ was determined by cytoarchitectural criteria (Knudsen, 1983). 

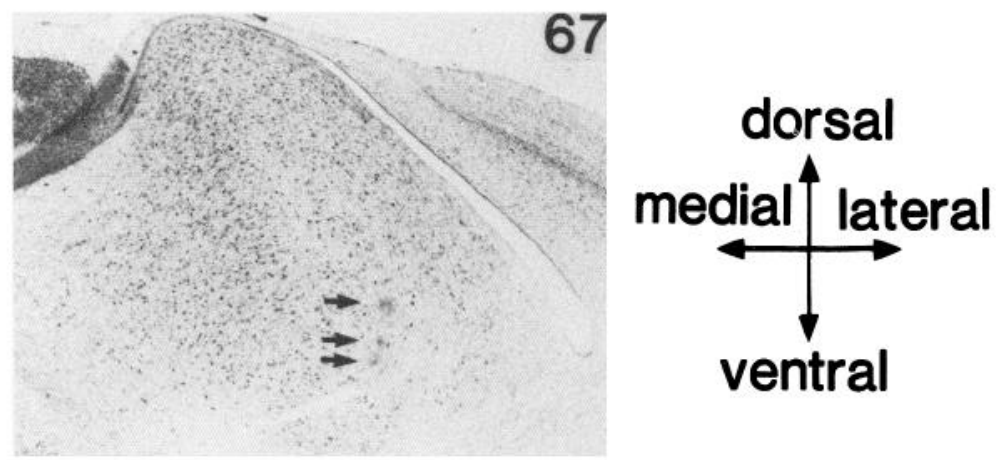

penetration number and position ( $\mathrm{mm}$ ) P1:0.75 post. 4.5 right

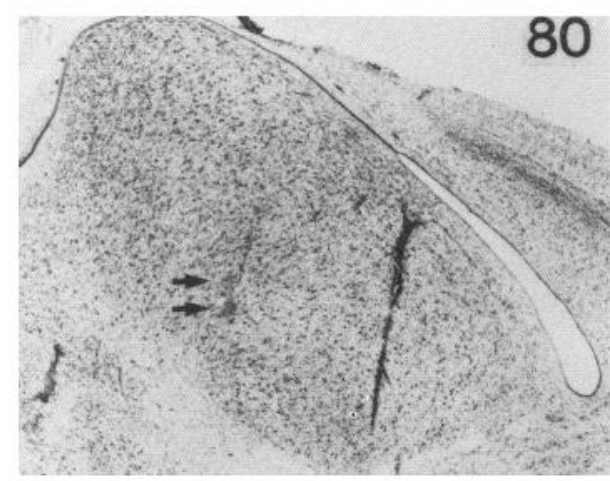

\section{P7: 0 post. 3.5 right}
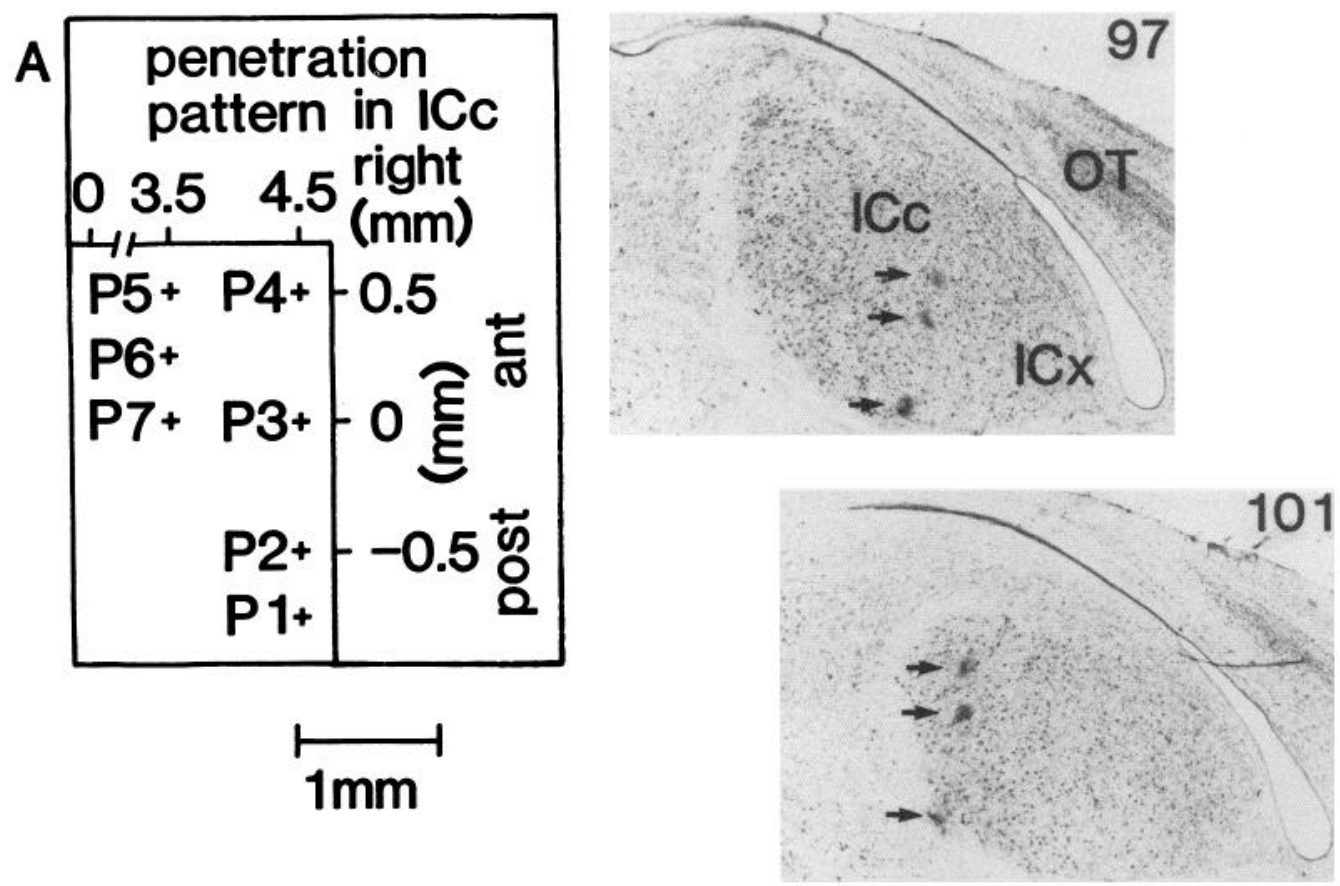

P4: 0.5 ant. 4.5 right

P5: 0.5 ant. 3.5 right

Figure 8. Systematic survey of the central nucleus. A, Schematic outline of the nucleus and penetrations P1-P7. The data are from the right central nucleus of one owl. Coordinate values refer to the distance from the reference post on the brain surface and not to the actual position, which is about $14 \mathrm{~mm}$ deep in the brain. $B$, Recovery of lesions in the same owl. Four penetrations were marked by specific lesion patterns. Coordinate values and penetration numbers are noted on the right side. Photographs of 4 sections with lesions at different anteroposterior coordinates (indicated by the arbitrary number of $30 \mu \mathrm{m}$ sections) show the position of these tracks in the nucleus. $P 1$ was found in sections $64-68 ; P 4$ in $95-98 ; P 5$ in $100-103$; and $P 7$ in 76-80. The heaviest labeling occurred in sections $65,96,101$, and 77 , respectively. The vertical scar in section 80 corresponds to the electrode track of $\mathrm{P} 3$. Higher numbers indicate more anterior sections. ICc, Central nucleus; $I C x$, external nucleus; $O T$, optic tectum.

array-specific ITD of P6 makes it seem probable that this penetration was made between P5 and P7, as intended.

When the array-specific ITDs from the 5 histologically identified penetrations are plotted along a straightened axis of the central nucleus, they increase linearly with position (Fig. 10). In Figure 7, we showed an anatomical map of ITD in the central nucleus. The data of Figure 10 physiologically demonstrate the existence of such a map. The physiological map extends further 


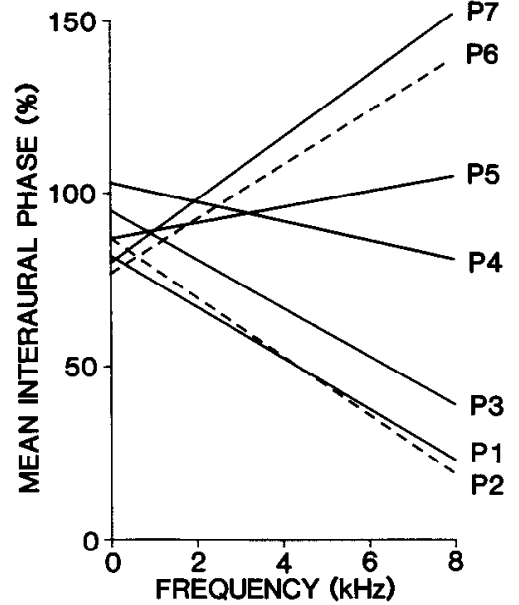

Figure 9. A map of ITD in the central nucleus. Data evaluated are ITD response curves obtained from the 7 penetrations shown in Figure 8 . The regression lines, indicating the array-specific ITDs (slope) and the relative discharge level at the array-specific ITDs ( $y$-intercepts), were calculated for each penetration as shown in Figures $1 B$ and $6 B$. Frequencies range from 2600 to $7500 \mathrm{~Hz}$. The regression lines belonging to $\mathrm{P} 2$ and $\mathrm{P} 6$ are shown with broken lines, because the positions of these penetrations could not be verified histologically. The mean square errors of all the arrays were significant.

to ipsilateral space than does the anatomical map. Arrays near the posterolateral margin of the central nucleus exhibit ITDs corresponding to the most peripheral contralateral azimuths. More anteriorly along the lateral margin, the array-specific ITDs correspond to more central loci in contralateral space. At the anterior margin of the central nucleus, the array-specific ITDs lie around zero. The entire ipsilateral space is represented along the medial margin of the central nucleus; array-specific ITDs increase as the electrode moves from anterior to posterior along the medial margin. The largest ipsilateral ITD in this owl is 91 $\mu \mathrm{sec}$. In other owls, array-specific ITDs up to $150 \mu \mathrm{sec}$ ipsilateral were found.

We have shown that mean interaural phase changes systematically along the tonotopic axis. We now test whether there is also a systematic change within one isofrequency lamina, as would be expected from the map of ITD shown in Figure 10. Mean interaural phase is plotted against position for 4 different isofrequency laminae in Figure 11. In each of these laminae (and in 3 additional ones, not plotted), mean interaural phase changes linearly with position (each individual correlation coefficient is significant at $p<0.05$ ). This demonstrates a map of mean interaural phase within each isofrequency lamina. If the data of all penetrations are considered, a 2-dimensional map of mean interaural phase becomes evident. This map is highly organized. The change of mean interaural phase with position, as revealed by the slope of the linear-regression lines, is smaller for low than for high frequencies $(0.19$ cycles $/ \mathrm{mm}$ for $3333 \mathrm{~Hz}$, 0.25 cycles $/ \mathrm{mm}$ for $4762 \mathrm{~Hz}, 0.32$ cycles $/ \mathrm{mm}$ for $6667 \mathrm{~Hz}$, and $0.35 \mathrm{cycles} / \mathrm{mm}$ for $8333 \mathrm{~Hz}$ ). The data obtained at 3 other isofrequency laminae show similar dependencies of mean interaural phase on position (slopes: 0.13 cycles $/ \mathrm{mm}$ for $3704 \mathrm{~Hz}$, 0.22 cycles $/ \mathrm{mm}$ for $4167 \mathrm{~Hz}, 0.25$ cycles $/ \mathrm{mm}$ for $5555 \mathrm{~Hz}$ ). Although the values of mean interaural phase in the isofrequency lamina of $8333 \mathrm{~Hz}$ change linearly with position, they are shifted relative to the others. Thercfore, the data of this lamina will not be considered in the following description.

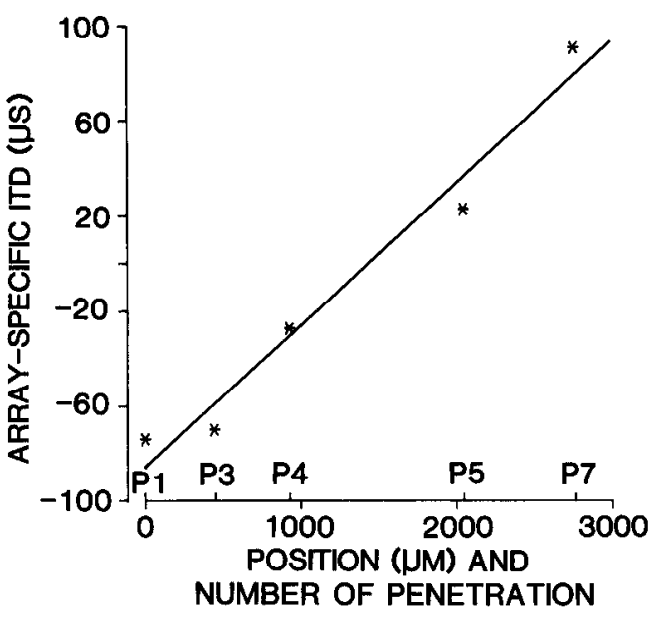

Figure 10. Array-specific ITDs change linearly with position. The linear correlation coefficient is $0.98(p<0.01)$. Data from P1, P3, P4, P5, and $\mathrm{P} 7$ are plotted. The position of the penetrations was calculated with reference to $\mathrm{P} 1$, using the sections with the heaviest labeling and the approximately $1 \mathrm{~mm}$ that separates the medial and lateral lesions. The $x$-axis plots the progression of the penetrations shown in Figure $8 \mathrm{~A}$ along a straight axis. In this way, the hooklike contour shown in Figure $8 A$ is unfolded by conserving the distances between penetrations.

The map of ITD can also be inferred from the 2-dimensional map of mean interaural phase shown in Figure 11. The regression lines intersect so that to the left of the intersection, lower frequencies have higher mean interaural phase values than do higher frequencies. The reverse is true to the right of the intersection. The greater the range of the mean interaural phase values in one penetration (imagine vertical lines in Fig. 11), the larger is the absolute value of the array-specific ITD, because, for a given frequency range, the slope of the regression line depends on the range of the mean interaural phase values (see Figs. $1 B$, $6 B$ ). At the intersection, the mean interaural phase values are equal. The slope of the regression line, and therefore the arrayspecific ITD at this locus, is zero. The sign of the ITD differs left and right of the intersection, because the slope is dependent on the sign of the gradient of mean interaural phase with frequency. The intersection in Figure 11 lies near a mean interaural phase of 0.9. This shows that the peak response of individual neurons contributes to the array-specific ITD (compare with Fig. $5 B$ ). Since all mean interaural phase values at the intersection point are near one and the array-specific ITD at this point is zero, the intercept of this particular array must also be zero. Note that this conclusion holds for all ITDs represented in the nucleus, because, owing to the common intersection and the linearity of the change of mean interaural phase with position, the $y$-intercept is nearly the same at each position. This relationship is also seen in the clustering of the $y$-intercepts of the regression lines around one in Figure 9. In conclusion, the 2-dimensional map of mean interaural phase covaries in one dimension with the frequency map to create a map of a third, derived, variable, a map of ITD.

\section{Tonotopy and the arrays}

The final experiment investigated the orientation of the ITD axis and the frequency axis with respect to each other. Figure 12 shows a coronal view of 2 isofrequency laminae in the central nucleus. Twenty-nine dorsoventral penetrations spaced $0.5 \mathrm{~mm}$ in a 2-dimensional grid covering all of the inferior colliculus 


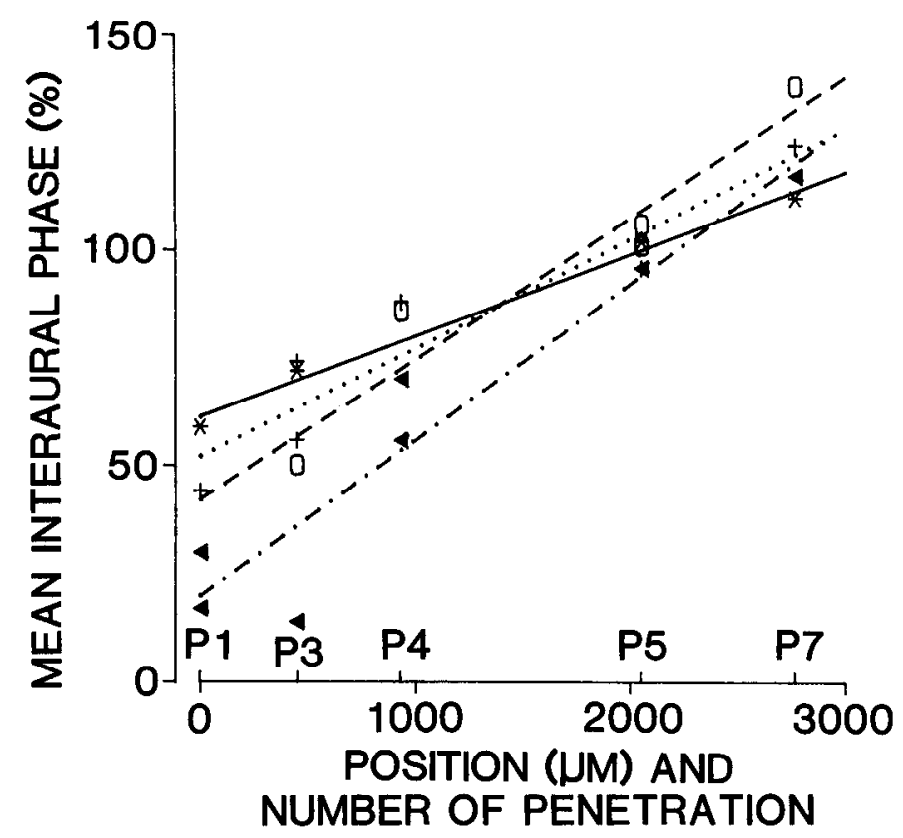

Figure 11. A map of mean interaural phase in each isofrequency lamina in the central nucleus. Data from P1, P3, P4, P5, and P7 are plotted. The $x$-axis is the same as in Figure 10. The stimulus was a tone burst at the best frequency of the recording site. Mean interaural phase was calculated as indicated in Figures $1 A$ and $6 A$. The data of the $3333 \mathrm{~Hz}$ $(*,-), 4763 \mathrm{~Hz}(+, \cdots), 6667 \mathrm{~Hz}(\mathrm{O},--)$, and $8333 \mathrm{~Hz}\left(4_{-},--\right)$ isofrequency laminae are plotted. Note the displacement of the data points and regression line of the $8333 \mathrm{~Hz}$ lamina with respect to the others.

were made in a single owl. Lesions were placed whenever a best frequency of 3000 or $6000 \mathrm{~Hz}$ was encountered. To assess the orientation of the arrays' axes, 2 of the penetrations were marked by many lesions. The distinct bands of lesions in Figure 12 demonstrate that the isofrequency laminae are flat slabs parallel to each other. The data, obtained at different anteroposterior coordinates, are plotted here onto one coronal section from the middle of the nucleus. This projection of all data onto a plane indirectly demonstrates the flatness of the isofrequency laminae: if the laminae were not flat, the 2 clusters would not be separated in space. From the distances of other frequencies relative to those of the marked laminae, it is clear that the other isofrequency laminae are similarly aligned. Note also that the penetrations werc madc nearly perpendicular to the isofrequency laminae. Measured on coronal sections, the penetration angle ranged from 75 to $90^{\circ}$ relative to the isofrequency laminae. The angle in the sagittal plane, which could be measured by rotating the computer image $90^{\circ}$, resulted in similar values. This result indicates that the ITD and frequency axes in the central nucleus are essentially perpendicular to each other.

\section{Discussion}

The analysis presented above shows a 2-dimensional map of interaural phase difference in the central nucleus. Interaural phase difference changes systematically, both within isofrequency laminae and perpendicular to them. The spatial relationship between the 2 parameters, interaural phase difference and frequency, underlies the extraction of a third parameter, ITD. The map of ITD consists of arrays of neurons oriented perpendicularly to isofrequency laminac. Retrograde transport of HRP from restricted loci in the external nucleus to arrays in the central

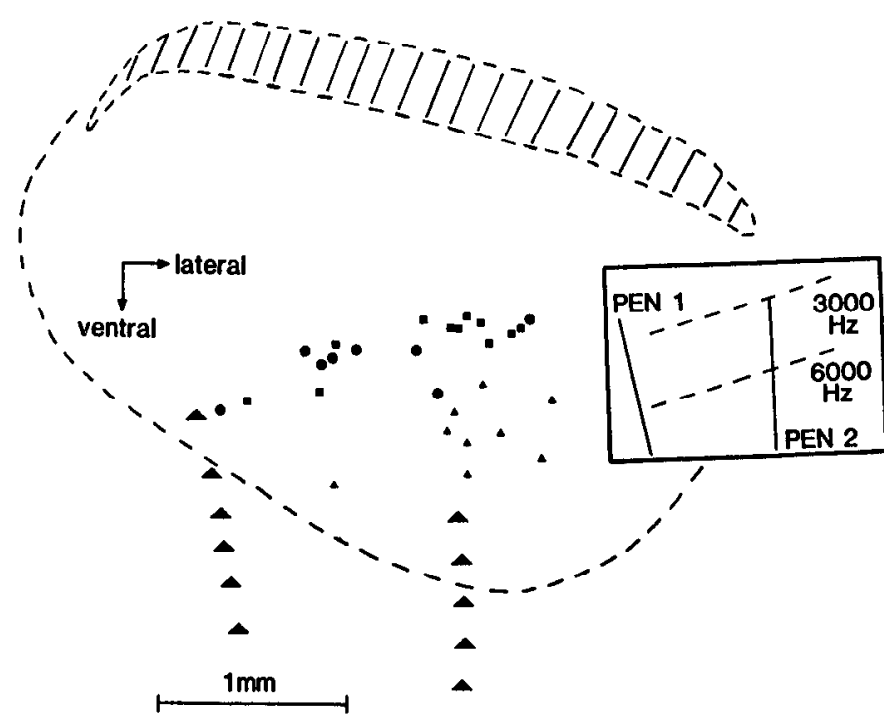

Figure 12. Angle between isofrequency laminae and 2 penetrations $(P E N)$. Twenty-nine penetrations were made in one owl in a grid with $0.5 \mathrm{~mm}$ spacing. Lesions were placed whenever a best frequency of 3000 or $6000 \mathrm{~Hz}$ was encountered. Two tracks were marked by many lesions $(\boldsymbol{A})$. The brain was cut after 2 reference holes were drilled into the brain perpendicular to the cutting plane. The border of the nucleus, the markers, lesions, and angle of the electrode tracks were digitized and stored in a computer. A 3-dimensional reconstruction was obtained with a computer system by visually aligning the scctions on a CRT monitor. Since $3000 \mathrm{~Hz}$ was always encountered above $6000 \mathrm{~Hz}$, and the precision of electrode positioning was better than $0.5 \mathrm{~mm}$, the correspondence between the isofrequency laminae was clear in sections where both lesions could be recovered. These lesions are marked by squares $(3000 \mathrm{~Hz})$ and triangles $(6000 \mathrm{~Hz})$, respectively. Lesions from sections with only a single one are marked by filled circles. Because of their location, these lesions also fall into one of the 2 clusters. The ventral line outlines the border of the inferior colliculus. The dorsal, hatched region indicates the tectal ventricle in a section from the middle of the nucleus, where the transected area was largest. The inset outlines the finding that isofrequency laminae are parallel slabs that were penetrated nearly perpendicularly.

nucleus establishes arrays as one of the inputs to space-specific neurons.

\section{Similarities between array-specific ITD and characteristic delay}

A dorsoventral array of neurons in the central nucleus spans a broad band of frequencies. Single neurons of an array have different best frequencies. There is only one ITD that excites all neurons of an array at the same relative response level, the arrayspecific ITD. The response at the array-specific ITD is, therefore, independent of frequency. Rose et al. (1966) stimulated single units at different frequencies and found that the response of some neurons was independent of frequency at one ITD. They called this ITD the "characteristic delay of the unit." By analogy, the array-specific ITD may also be called the "characteristic delay of the array."

\section{Possible functional significance of the arrays}

In Figure 13, a schematic model of the ITD-sensitive region of the central nucleus is shown. It is a 2-dimensional map of interaural phase difference that covaries with frequency along the dorsoventral axis. Interaural phase difference also changes systematically within each isofrequency band. In this way, a map of ITD is created in the anteroposterior dimension. 
Suppose an owl receives a noise stimulus having an ITD of $50 \mu \mathrm{sec}$. Two neurons in the frequency band of $5000 \mathrm{~Hz}$, for example, will be maximally excited-one representing an interaural phase difference of $25 \%$, and one that represents an interaural phase difference of $-75 \%$. The same 2 neurons will also be maximally excited to stimulation with noise having an ITD of $-150 \mu \mathrm{sec}$ because individual neurons cannot distinguish noises having ITDs differing by integral numbers of periods of their best frequencies. In contrast, the response of the columns or arrays in the model is unambiguous: at any ITD, only one array is maximally excited and at different ITDs, different arrays are maximally excited. Therefore, the array has properties that are absent in a single unit. It can unambiguously represent an ITD and signal this ITD to the next neural station, where it can be deciphered. Even if all individual neurons forming an array had a characteristic delay, which was the same for all neurons of an array, the individual neurons could not signal their characteristic delay, and no unique ITD could be assigned to the signal of individual neurons at the next neural station because these neurons show phase ambiguity.

In space-specific neurons, more than one frequency is necessary to resolve phasc ambiguity (Takahashi and Konishi, 1986). The broad frequency band encompassed by the array fulfills this condition and, therefore, provides a basis for the resolution of phase ambiguity in the array's target neuron in the space map. The direct projection to the space-specific neurons constitutes further evidence that an array forms a functional unit. The HRP experiments show that the arrays probably signal their arrayspecific ITD to the space-specific neurons to which they project. Knudsen $(1983,1984)$ studied the connection between the central and the external nuclei by means of retrograde labeling. He found a converging input from many isofrequency laminae in the central nucleus onto one spot in the external nucleus, and an anteroposterior gradient similar to ours [notice that in Figure 10 of Knudsen (1983), only the anterior third of the inferior colliculus is shown, and that no cells were labeled in the posterior part (E. Knudsen, personal communication)].

\section{Comparison between the maps in the external nucleus and in the central nucleus}

In the external nucleus, the location of a stimulus is mapped topographically (Knudsen and Konishi, 1977, 1978). This map of auditory space requires 2 dimensions of the nucleus: ITD or azimuth, varying roughly along the anteroposterior axis, and interaural intensity difference (IID) or elevation along the dorsoventral axis. Any given coordinate in this 2-dimensional grid corresponds to a unique pair of ITD and IID (Moiseff and Konishi, 1981). In the central nucleus, the 2 corresponding dimensions are occupied by interaural phase difference. In addition, frequency also varies along the dorsoventral axis. In both the external and the central nucleus, the mapping along the third dimension is unknown. In the central nucleus, the representation of a single ITD requires one dimension and the map of ITD occupies the other one. One transformation from the central to the external nucleus is the convergence of frequency channels (Knudsen, 1983, 1984). This convergence has 2 effects: first, it underlies the resolution of phase ambiguity in space-specific neurons (Takahashi and Konishi, 1986); and second it leads to a loss of tonotopy (Knudsen and Konishi, 1978), and thus "frees" one of the dimensions in the external nucleus. The map of IID occupies this "freed" dimension and the map of auditory space emerges.

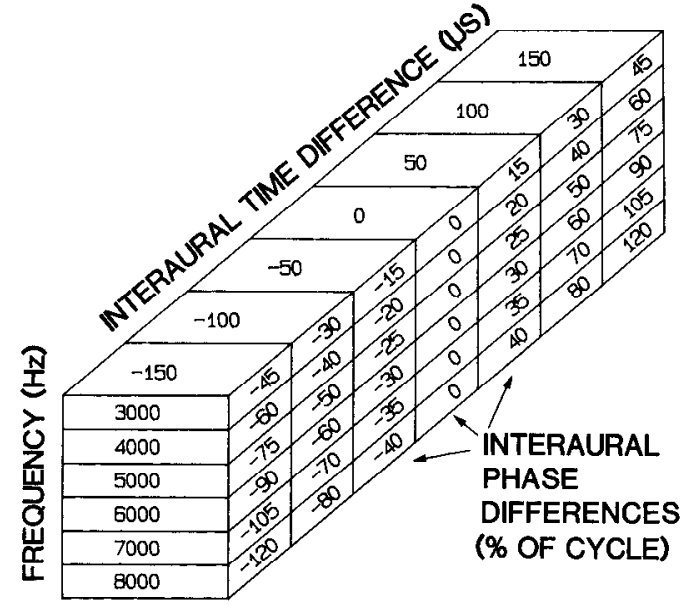

Figure 13. Schematic model of ITD-sensitive region of the central nucleus. The mediolateral axis runs from left to right, the dorsoventral axis from top to bottom. Note that the inclined axis of this figure corresponds to the straightened $x$-axes of Figures 10 and 11 , and not to the actual anteroposterior axis of the owl (Fig. 8A). The slabs of discrete values in this scheme do not imply a compartmentalization in the central nucleus. Interaural phase difference forms a 2-dimensional map that covaries along one dimension with the tonotopic order to create the map of a third, derived variable, ITD, which runs perpendicular to the frequency axis.

In the central nucleus, the entire ITD range of the barn owl is mapped, whereas in the external nucleus, ITDs corresponding to eccentric ipsilateral loci are not represented (Knudsen and Konishi, 1977, 1978). The meaning of this difference is not yet understood. Interestingly, however, the anatomical map in the central nucleus essentially parallels the map in the external nucleus: we did not find clusters of heavy labeling in the medial part of the central nucleus, which represents ipsilateral space. The parts of the anatomical and physiological maps in the central nucleus that correspond to contralateral and frontal space (Figs. 7, 10) are similar. A quantitative comparison is difficult because of the few data points and the curvature of the ITD axis. We have shown in Figures 10 and 11 that a linear regression fits the maps in the central nucleus. In the external nucleus, the frontal auditory space is overrepresented (Knudsen and Konishi, 1977). This might also be the case in the central nucleus, but many more data points will be needed in order to discriminate between an apparent linearity and a possible nonlinearity. It is interesting that during the mapping of both the external nucleus (Knudsen and Konishi, 1977, 1978) and the central nucleus (this study), only interaural delays corresponding to azimuths smaller than $50^{\circ}$ were found. Nevertheless, in recordings from single space-specific neurons (unpublished observations) and arrays (Fig. 5), we found ITDs covering the entire physiological ITD range of the barn owl. This could mean that the most peripheral locations are represented within a small portion of the nucleus.

We have demonstrated a gradient of ITD in the central nucleus, but we do not intend to present the exact quantitative relationship between position and ITD, because there are many sources of variability in this derived map, for example, errors in measuring mean interaural phase and best frequency. It is also possible that the units forming an array sometimes do not lic along a straight linc. Espccially at the low- and high-frcquency ends, we sometimes found mean interaural phase values that 
were displaced from the straight line formed by the rest of the units (best frequencies between 2500 and $7500 \mathrm{~Hz}$ ). For this reason we did not include units with best frequencies greater than $7500 \mathrm{~Hz}$ in Figures 9 and 10.

\section{Creation of a 2-dimensional map of interaural phase difference}

The central nucleus contains a very systematic 2 -dimensional map of interaural phase difference. We know the quantitative relationship within this map much better than that within the map of ITD, because we directly measured the values of the interaural phase difference within predetermined isofrequency laminae in a spatial grid revealed by lesions. How is the lattice of Figure 13 generated? The problem may be divided in two: the map of interaural phase difference within isofrequency laminae and the map of interaural phase difference perpendicular to isofrequency laminae.

We first discuss how the map within the isofrequency laminae may be created. Recording of evoked potentials in nucleus laminaris, the first site of binaural convergence in the barn owl, suggests a map of interaural phase difference within each isofrequency lamina in this nucleus (Sullivan and Konishi, 1986). Thus, the map of interaural phase difference within isofrequency laminae in the central nucleus could be created by topologically conservative projections from nucleus laminaris. It seems possible that the solution of the second problem mentioned above also lies in nucleus laminaris and that, therefore, the 2-dimensional map in the central nucleus may derive from a similar map in nucleus laminaris.

\section{Comparison with other systems}

Many concepts of sound localization and binaural interaction have origins in studies of mammalian systems such as those of humans (for review, see Hafter, 1984), the cat (for review, see Yin and Kuwada, 1984), the dog (Goldberg and Brown, 1969), or the kangaroo rat (Stillman, 1971). Mammals, in contrast to the barn owl, use interaural intensity and interaural time difference for coding azimuth (duplex theory of sound localization; for review, see Hafter, 1984). So far, the barn owl is the only animal known to use interaural phase differcnces derived from carrier frequencies greater than $5 \mathrm{kHz}$ (Moiseff and Konishi, 1981; Sullivan and Konishi, 1984). The mammalian inferior colliculus is tonotopically organized (Rose et al., 1963; Merzenich and Reid, 1974; Semple and Aitkin, 1979), as is the central nucleus of the barn owl. Interestingly, Yin et al. (1983) reported preliminary data showing a gradient of characteristic delay in the cat's inferior colliculus. With free-field stimulation, Aitkin et al. (1985) found azimuth-selective units in the lowfrequency region of the cat's inferior colliculus. The arrangement of the neurons in one isofrequency region $(1.3 \mathrm{kHz})$ suggests a gradient of azimuth. The selectivity of these neurons for azimuth is presumably based on ITDs. Thus, this map is similar to the map of mean interaural phase within one isofrequency lamina reported here. Aitkin et al. (1985) did not present sufficient data to show maps of azimuth and their alignment in other isofrequency laminac. Thercfore, it is not clear at the moment whether the cat also uses a broad band of frequencies for localization of azimuth by ITD. It seems possible that the principles of auditory processing demonstrated in the barn owl, such as the organization of phase-sensitive units into arrays and the orthogonal mapping of frequency and ITD, may also be of relevance to the understanding of the mammalian auditory system.

\section{Reliability of measuring characteristic delay}

When Rose et al. (1966) introduced characteristic delay as a neural correlate for representing ITD, they assumed that characteristic delay is a result of an anatomically imposed constant delay. This concept and interpretation have been widely accepted (Yin and Kuwada, 1984; Konishi, 1986; Konishi et al., 1987).

In 1983, Yin and Kuwada introduced a method to calculate characteristic delay: Characteristic delay corresponds to the slope of the linear regression line calculated from a plot of different mean interaural phase values versus stimulus frequencies. The measures of characteristic delay (slope) and characteristic phase ( $y$-intercept) are, however, sensitive to slight variabilities in mean interaural phase (see data of Yin and Kuwada, 1983). These authors themselves expressed caution about the usefulness of their method for obtaining information about the ITD of a freefield source. We argue that caution is especially necessary in interpreting values from units with a narrow, V-shaped tuning curve, like those in the central nucleus. A unit is stimulated at its best frequency and also at other frequencies within its excitatory tuning curve. First, owing to the narrow bandwidth of the neurons, the number of significant data points (separated in period by about the circular standard deviation) in phase/frequency plots are limited. Also, the narrower the bandwidth, the more sensitive are the quantified parameters to slight variabilities. There may be small changes in the response of the unit at nonoptimal frequencies that may cause shifts of mean interaural phase. Changes of mean interaural phase that depend on stimulus intensity, for example, have been reported ( $Y$ in and $\mathrm{Ku}$ wada, 1983). Furthermore, it is not clear how the contribution of each frequency should be weighted. Correction for spike number would not be enough, because the center point, which usually represents the best frequency, has the least influence on the slope of the regression line.

Yin and Kuwada (1983) concluded from their study on single units that the composite peak (see Materials and Methods) and not the characteristic delay may be the important parameter for signaling information about the ITD of a free-field source. The composite peak is less influenced by slight changes of the response that possibly occur at nonoptimal frequencies. We found that the mean absolute difference between the array-specific ITD and the characteristic delay of a unit was $84 \mu \mathrm{sec}$ for 7 examples. The mean absolute difference between the composite peaks of the single units and the array-specific ITD is smaller (28 $\mu \mathrm{sec}$; Wilcoxon matched-pairs signed-rank test; $n=7 ; p<0.05)$. This also suggests that the composite peak may tell more about the functional significance of a unit than does the characteristic delay. In general, phase/frequency plots may be appropriate for the analysis of the arrays and for units with wide, U-shaped tuning curves, but not for units with narrow, V-shaped tuning curves. In units with U-shaped tuning curves and the arrays, many of the above-mentioned problems (number of points, intensity) do not arise.

\section{References}

Aitkin, L. M., J. D. Pettigrew, M. B. Calford, S. C. Phillips, and L. Z. Wise (1985) Representation of stimulus azimuth by low-frequency neurons in inferior colliculus of the cat. J. Neurophysiol. 53: 43-59. Batschelet, E. (1981) Circular Statistics in Biology, Academic, London. Geisler, C. D., W. S. Rhode, and D. W. Hazelton (1969) Response of inferior colliculus neurons in the cat to binaural acoustic stimuli having wide-band spectra. J. Neurophysiol. 32: 960-974. 
Goldberg, J. M., and P. B. Brown (1969) Response of binaural neurons of dog superior olivary complex to dichotic tonal stimuli: Some physiological mechanisms of sound localization. J. Neurophysiol 32:613636.

Hafter, E. (1984) Spatial hearing and the duplex theory: How viable is the model? In Dynamic Aspects of Neocortical Function, G. M. Edelman, W. E. Gall, and W. M. Cowan, eds., pp. 425-448, Wiley, New York.

Kiang, N. Y.S. (1965) Discharge patterns of single fibers in the cat's auditory nerve. Research Monograph No. 35, M.I.T. Press, Cambridge, MA.

Knudsen, E. (1983) Subdivisions of the inferior colliculus in the barn owl (Tyto alba). J. Comp. Neurol. 218: 174-186.

Knudsen, E. (1984) Synthesis of a neural map of auditory space in the owl. In Dynamic Aspects of Neocortical Function, G. M. Edelman, W. E. Gall, and W. M. Cowan, eds., pp. 375-396, Wiley, New York.

Knudsen, E. I., and M. Konishi (1977) A neural map of auditory space in the owl. Science 200: 795-797.

Knudsen, E. I., and M. Konishi (1978) Space and frequency are represented separately in auditory midbrain of the owl. J. Neurophysiol. 41: 870-884.

Konishi, M. (1973) How the owl tracks its prey. Am. Sci. 61:414 424.

Konishi, M. (1986) Centrally synthesized maps of sensory space. Trends Neurosci. 9: 163-168.

Konishi, M., T. Takahashi, H. Wagner, W. E. Sullivan, and C. E. Carr (1987) Neurophysiological and anatomical substrates of sound localization in the owl. In Functions of the Auditory System, G. M. Edelman, W. E. Gall, and W. M. Cowan, eds., Wiley, New York (in press).

Merzenich, M. M., and M. D. Reid (1974) Representation of the cochlea within the inferior colliculus of the cat. Brain Res. 77: 397415 .

Mesulam, M. M. (1978) Tetramethyl benzidine for horseradish peroxidase neurochemistry: A non-carcinogenic blue reaction product with superior sensitivity for visualizing neural afferents and efferents. J. Histochem. Cytochem. 26: 106-117.

Moiseff, A., and M. Konishi (1981) Neuronal and behavioral sensitivity to binaural time differences in the owl. J. Neurosci. 1: 40-48.

Rose, J. E., D. O. Greenwood, J. M. Goldberg, and J. E. Hind (1963) Some discharge characteristics of single neurons in the inferior colliculus of the cat. I. Tonotopic organization, relation of spike-counts to tone intensity, and firing patterns of single elements. J. Neurophysiol. 26: 294-320.

Rose, J. E., N. G. Grass, C. D. Geisler, and J. E. Hind (1966) Some neural mechanisms in the inferior colliculus of the cat which may be relevant to localization of a sound source. J. Neurophysiol. 29: 288314.

Roth, G. L., L. M. Aitkin, R. A. Anderson, and M. Merzenich (1978) Some features of the spatial organization of the central nucleus of the inferior colliculus of the cat. J. Comp. Neurol. 182: 661-680.

Semple, M. N., and L. M. Aitkin (1979) Representation of sound frequency and laterality by units in central nucleus of cat inferior colliculus. J. Neurophysiol. 42: 1626-1639.

Stillman, R. D. (1971) Characteristic delay neurons in the inferior colliculus of the kangaroo rat. Exp. Neurol. 32: 404-412.

Sullivan, W. E., and M. Konishi (1984) Segregation of stimulus phase and intensity coding in the cochlear nucleus of the barn owl. J. Neurosci. 4: 1787-1799.

Sullivan, W. E., and M. Konishi (1986) Neural map of interaural phase difference in the owl's brainstem. Proc. Natl. Acad. Sci. USA 83 . $8400-8404$.

Takahashi, T., and M. Konishi (1983) Functional organization of the barn owl's inferior colliculus. Soc. Neurosci. Abstr. 9: 212.

Takahashi, T., and M. Konishi (1986) Selectivity for interaural time difference in the owl's midbrain. J. Neurosci. 6: 3413-3422.

Takahashi, T., A. Moiseff, and M. Konishi (1984) Time and intensity cues are processed independently in the auditory system of the owl. J. Neurosci. 4: 1781-1786.

Yin, T. C., and S. Kuwada (1983) Binaural interaction in low-frequency neurons in inferior colliculus of the cat. III. Effects of changing frequency. J. Neurophysiol. 50: 1020-1042.

Yin, T. C., and S. Kuwada (1984) Neuronal mechanisms of binaural interactions. In Dynamic Aspects of Neocortical Function, G. M. Edelman, W. E. Gall, and W. M. Cowan, eds., pp. 263-313, Wiley, New York.

Yin, T. C., J. C. K. Chan, and S. Kuwada (1983) Characteristic delays and their topographical distribution in the inferior colliculus of the cat. In Mechanisms of Hearing, W. R. Webster and L. M. Aitkin, eds., pp. 94-99, Monash U. P., Clayton, Victoria.

Yin, T. C., J. C. K. Chan, and D. R. Irvine (1986) Effects of interaural time delays of noise stimuli on low frequency cells in the cat's inferior colliculus. I. Responses to wide-band noise. J. Neurophysiol. 55:280300 . 